\title{
Inhibition of endogenous thioredoxin in the heart increases oxidative stress and cardiac hypertrophy
}

\author{
Mitsutaka Yamamoto, ${ }^{1}$ Guiping Yang, ${ }^{1}$ Chull Hong, ${ }^{1}$ Jing Liu, ${ }^{1}$ Eric Holle, ${ }^{2}$ \\ Xianzhong $\mathrm{Yu},{ }^{2}$ Thomas Wagner, ${ }^{2}$ Stephen F. Vatner, ${ }^{1}$ and Junichi Sadoshima ${ }^{1}$ \\ ${ }^{1}$ Cardiovascular Research Institute, Department of Cell Biology and Molecular Medicine and Department of Medicine, \\ University of Medicine and Dentistry of New Jersey, New Jersey Medical School, Newark, New Jersey, USA \\ ${ }^{2}$ Oncology Research Institute, Greenville, South Carolina, USA
}

\begin{abstract}
Thioredoxin $1(\operatorname{Trx} 1)$ has redox-sensitive cysteine residues and acts as an antioxidant in cells. However, the extent of Trx1 contribution to overall antioxidant mechanisms is unknown in any organs. We generated transgenic mice with cardiac-specific overexpression of a dominant negative (DN) mutant (C32S/C35S) of Trx1 (Tg-DN-Trx1 mice), in which the activity of endogenous Trx was diminished. Markers of oxidative stress were significantly increased in hearts from Tg-DN-Trx1 mice compared with those from nontransgenic (NTg) mice. Tg-DN-Trx1 mice exhibited cardiac hypertrophy with maintained cardiac function at baseline. Intraperitoneal injection of $\mathrm{N}$-2-mercaptopropionyl glycine, an antioxidant, normalized cardiac hypertrophy in Tg-DN-Trx1 mice. Thoracic aortic banding caused greater increases in myocardial oxidative stress and enhanced hypertrophy in Tg-DN-Trx1 compared with NTg mice. In contrast, transgenic mice with cardiac-specific overexpression of wild-type Trx1 did not show cardiac hypertrophy at baseline but exhibited reduced levels of hypertrophy and oxidative stress in response to pressure overload. These results demonstrate that endogenous Trx 1 is an essential component of the cellular antioxidant mechanisms and plays a critical role in regulating oxidative stress in the heart in vivo. Furthermore, inhibition of endogenous $\operatorname{Trx} 1$ in the heart primarily stimulates hypertrophy, both under basal conditions and in response to pressure overload through redox-sensitive mechanisms.
\end{abstract}

J. Clin. Invest. 112:1395-1406 (2003). doi:10.1172/JCI200317700.

\section{Introduction}

Accumulating evidence suggests that oxidative stress plays an important role in mediating pathologic responses in cardiac myocytes and various diseases in the heart (reviewed in refs. 1,2). Production of reactive oxy-

Received for publication December 30, 2002, and accepted in revised form September 9, 2003.

Address correspondence to: Junichi Sadoshima, Cardiovascular Research Institute, University of Medicine and Dentistry of New Jersey, New Jersey Medical School, 185 South Orange Avenue, Medical Science Building G-609, Newark, New Jersey 07103, USA. Phone: (973) 972-8619; Fax: (973) 972-8919;

E-mail: Sadoshju@umdnj.edu.

A preliminary form of this work was presented at the LateBreaking Science Session of the American Heart Association Scientific Sessions 2002 in Chicago, Illinois, USA, on November 17,2002

Conflict of interest: The authors have declared that no conflict of interest exists.

Nonstandard abbreviations used: superoxide dismutase (SOD); manganese SOD (MnSOD); copper zinc SOD (CuZnSOD); thioredoxin (Trx); human $\operatorname{Trx} 1(\mathrm{~h} \operatorname{Tr} \mathrm{x} 1)$; dominant negative hTrx1 (DN-hTrx1); DN-hTrx1 transgenic (Tg-DN-Trx1); wildtype hTrx1 transgenic (Tg-Trx1); malondialdehyde (MDA); 4-hydroxyalkenals (4-HAE); reduced glutathione (GSH); oxidized glutathione (GSSG); atrial natriuretic factor (ANF); left ventricle/left ventricular ( $L V) ; L V$ end-diastolic diameter (LVEDD); LV end-systolic diameter (LVESD); LV ejection fraction (LVEF); percent fractional shortening (\%FS); 8-hydroxy-2'deoxyguanosine (8-OHdG); nontransgenic (NTg);

$\mathrm{N}$-2-mercaptopropionyl glycine (MPG); LV weight/body weight ratio (LVW/BW); LV weight/tibial length ratio (LVW/TL); extracellular signal-regulated kinase (ERK); vitamin D3-upregulated protein-1 (VDUP1). gen species by mitochondrial sources as well as nonphagocytic oxidases is enhanced in various pathologic stimuli in the heart $(1,2)$. To counteract increased levels of oxidative stress, cells have antioxidant mechanisms that include superoxide dismutases (SODs) - which are present in mitochondria, such as manganese SOD (MnSOD), cytosol, such as copper zinc SOD (CuZnSOD), and plasma membrane and extracellular spaces (such as extracellular SOD) - catalase, glutathione peroxidases, peroxiredoxins, and thioredoxin $(\operatorname{Trx})(1,2)$.

$\operatorname{Tr} x 1$ is a $12-\mathrm{kDa}$ multifunctional protein with a redoxactive disulfide/dithiol within the conserved active site ${ }^{32}$ Cys-Gly-Pro- ${ }^{35}$ Cys (reviewed in refs. 3, 4). Trx1, Trx reductase, and NADPH, collectively called the Trx system, operate as a powerful protein-disulfide oxidoreductase system (3, 4). Trx1 seems unique among antioxidants in that it interacts directly with various intracellular-signaling molecules as well as transcription factors, thereby affecting cell growth and cell survival in some cell types $(3,4)$. Recent evidence suggests that either protein expression or the activity of Trx 1 is modified in some cardiovascular diseases (5-8). Surprisingly, however, the importance of endogenous $\operatorname{Trx} 1$ as an antioxidant and the in vivo role of $\operatorname{Tr} x 1$ in cell growth responses are not clearly understood in any organs, including the heart.

We hypothesized that endogenous Trx1 plays an important role in regulating the tissue level of oxidative stress, thereby controlling cardiac myocyte growth responses. Although mice systemically deficient in Trx1 have been generated by homologous recombination, 
they are embryonic lethal (9). In order to examine the role of endogenous Trx 1 in the heart in vivo, we generated transgenic mice with cardiac-specific overexpression of dominant negative human Trx1 (DN-hTrx1), in which the disulfide oxidoreductase activity of Trx is selectively diminished among cellular antioxidant mechanisms. We examined whether inhibition of endogenous Trx1 increases tissue levels of oxidative stress and whether it affects any cardiac phenotype, including cardiac hypertrophy, under basal conditions as well as in response to pressure overload.

\section{Methods}

Transgenic mice. DN-hTrx1 was generated by mutation of ${ }^{32} \mathrm{Cys}$ and ${ }^{35} \mathrm{Cys}$ of hTrx1 to Ser using QuikChange (Stratagene, La Jolla, California, USA). This redoxinactive mutant of $\operatorname{Tr} x 1$ has been shown to work as a dominant negative for endogenous $\operatorname{Trx} 1$ in a breast cancer cell line (10). DN-hTrx1 transgenic mice (hereafter designated as Tg-DN-Trx1) as well as wild-type hTrx1 mice (hereafter designated as Tg-Trx1) were generated on an FVB background using the $\alpha$-myosin heavy chain promoter (courtesy of J. Robbins, University of Cincinnati, Cincinnati, Ohio, USA) to achieve cardiac-specific expression.

Immunoblot analyses. Tissue homogenates were prepared in buffer $\mathrm{A}$, containing $150 \mathrm{mM} \mathrm{NaCl}, 50 \mathrm{mM}$ Tris $(\mathrm{pH}$ 7.5), $1 \%$ Triton $\mathrm{X}-100,10 \%$ glycerol, 5 mM EDTA, $1 \mathrm{mM}$ $\mathrm{Na}_{3} \mathrm{VO}_{4}, 10 \mathrm{mM} \mathrm{NaF}, 0.5 \mathrm{mM}$ 4-(2-aminoethyl)benzenesulfonyl fluoride hydrochloride, $0.5 \mu \mathrm{g} / \mathrm{ml}$ aprotinin, and $0.5 \mu \mathrm{g} / \mathrm{ml}$ leupeptin. We used anti-hTrx $1 \mathrm{mAb}$ (clones 2G11 and 4H9; BD Pharmingen, San Diego, California, USA), anti-CuZnSOD Ab (BD Pharmingen), antiMnSOD Ab (Upstate Biotechnology Inc., Lake Placid, New York, USA), and anti-catalase Ab (Abcam Ltd., Cambridge, United Kingdom) as primary Ab's. All anti-phosphospecific and corresponding non-phosphospecific Ab's against protein kinases were obtained from Cell Signaling Technology Inc. (Beverly, Massachusetts, USA).

Detection of oxidative stress and antioxidant mechanisms. Tissue homogenates were prepared using $20 \mathrm{mM}$ phosphate buffer ( $\mathrm{pH}$ 7.4) with $5 \mathrm{mM}$ butylated hydroxytoluene. Tissue levels of malondialdehyde (MDA) and 4-hydroxyalkenals (4-HAE) were determined using a Bioxytech LPO-586 kit (Oxis International Inc., Portland, Oregon, USA) (11). The tissue level of reduced glutathione/oxidized glutathione (GSH/GSSG) was determined using a Bioxytech GSH/GSSG-412 kit (Oxis International Inc.). For measurement of GSSG, the thiol-scavenging reagent 1-methyl-2-vinylpyridium trifluoromethanesulfonate was included in the homogenization buffer to minimize oxidation of GSH to GSSG during sample preparation, and only fresh samples were used (12).

RT-PCR. Total RNA was prepared using TRIzol (Invitrogen Corp., Carlsbad, California, USA) and then subjected to RT-PCR using the First-Strand cDNA Synthesis kit (Invitrogen Corp.) as previously described (13). The following oligonucleotide primers specific for mouse car- diac genes were used in this study: atrial natriuretic factor (ANF), sense $5^{\prime}$-ATGGGCTCCTTCTCCATCAC-3' and antisense $5^{\prime}$-TCTTCGGTACCGGAAGCT- $3^{\prime}$; $\alpha$-skeletal actin, sense $5^{\prime}$-TATTCCTTCGTGACCACAGCTGAACGT- $3^{\prime}$ and antisense 5'-CGCGAACGCAGACGCGAGTGCGC-3'; and GAPDH, $5^{\prime}$-TTCTTGTGCAGTGCCAGCCTCGTC- $3^{\prime}$ and antisense $5^{\prime}$-TAGGAACAGGGAAGG-CCATGCCAG- ${ }^{\prime}$. We also used oligonucleotide primers common to mouse and human Trx1, sense 5'-GGTGTGGACCTTGCAAAATGATC-3' and antisense 5'-GGCTTCAAGCTTTTCCTT-3'.

Insulin reduction assay for $\operatorname{Tr} x$. The activity of $\operatorname{Trx}$ in the heart was determined by the insulin reduction assay, according to the method described by Holmgren and Bjornstedt with a slight modification (14). Hearts were homogenated with ice-cold PBS containing $0.5 \mathrm{mM} 4-$ (2-aminoethyl)benzenesulfonyl fluoride hydrochloride, $0.5 \mu \mathrm{g} / \mathrm{ml}$ aprotinin, and $0.5 \mu \mathrm{g} / \mathrm{ml}$ leupeptin. An equal amount of protein $(50 \mu \mathrm{g})$ in a volume of $8 \mu \mathrm{l}$ was preincubated with $2 \mu \mathrm{l}$ of the DTT activation buffer $(100 \mathrm{mM}$ Tris-Cl [pH 7.5], $2 \mathrm{mM}$ EDTA, $1 \mathrm{mg} / \mathrm{ml} \mathrm{BSA}$, and $2 \mathrm{mM}$ DTT) at $37^{\circ} \mathrm{C}$ for 15 minutes. The samples were then mixed with $110 \mu$ l of reaction mixture $(100 \mathrm{mM}$ Tris-Cl [pH 7.5], 2.0 mM EDTA, $0.2 \mathrm{mM} \mathrm{NADPH}, 1.0 \mu \mathrm{g}$ human Trx reductase [American Diagnostica Inc., Greenwich, Connecticut, USA], and $140 \mu \mathrm{M}$ insulin) and incubated at $25^{\circ} \mathrm{C}$. Reduction in absorbance at $340 \mathrm{~nm}$, indicating oxidation of NADPH, was measured using a spectrophotometer at 30-second intervals. As a control, the samples were mixed with the reaction mixture without insulin. Changes in absorbance in the absence of insulin were subtracted from those in the presence of insulin.

Echocardiography. Mice were anesthetized and echocardiography was performed using ultrasonography (Acuson Sequoia C256, Siemens Medical Solutions USA Inc., Malvern, Pennsylvania, USA) as previously described (15). A $13-\mathrm{MHz}$ linear ultrasound transducer was used. M-mode measurements of left ventricular $(\mathrm{LV})$ internal diameter were taken from more than three beats and averaged. LV end-diastolic diameter (LVEDD) was measured at the time of the apparent maximal LV diastolic dimension, while LV end-systolic diameter (LVESD) was measured at the time of the most anterior systolic excursion of the posterior wall. LV ejection fraction (LVEF) and percent fractional shortening $(\% \mathrm{FS})$ were calculated as follows: $\mathrm{LVEF}=\left[(\mathrm{LVEDD})^{3}-(\mathrm{LVESD})^{3}\right] /(\mathrm{LVEDD})^{3}$; $\% \mathrm{FS}=($ LVEDD - LVESD $) /$ LVEDD $\times 100$.

Histological analyses. The LV accompanied by the septum was cut into base, midportion, and apex, fixed with $10 \%$ formalin, embedded in paraffin, and sectioned at $6 \mu \mathrm{m}$ thickness. The sections were incubated in $3 \% \mathrm{H}_{2} \mathrm{O}_{2}$ in PBS to prevent endogenous peroxidation and blocked with 5\% BSA in PBS. Anti-8-hydroxy-2'deoxyguanosine (anti-8-OHdG) Ab (Oxis International Inc.) was diluted to $7.5 \mu \mathrm{g} / \mathrm{ml}$ in PBS and applied to the sections for 1 hour at $37^{\circ} \mathrm{C}$. After washing, biotinylated secondary $\mathrm{Ab}$ (anti-mouse IgG; BD Pharmingen) was applied for 1 hour, followed by streptavidin-HRP (BD Pharmingen) for 30 minutes at room temperature. 


\section{Figure 1}

(a) Heart homogenates were prepared from Tg-DN-Trx1 and NTg mice. Immunoblot analyses were conducted using anti-hTrx1 Ab. Short (15-second) and long (5minute) exposures of the immunoblot are shown. After long exposure, endogenous mouse Trx 1 was detected. Note that the anti-hTrx1 $\mathrm{Ab}$ (clone 2G11) does not detect mouse $\operatorname{Tr} x 1$ as efficiently as it detects hTrx1. (b) Tissue homogenates were prepared from various organs. Immunoblot analyses were conducted using anti-hTrx1 Ab. (c) RT-PCR analyses of Trx 1 and GAPDH. Total RNA was extracted from Tg-DN-Trx1 (line no. 13) and NTg mice. The lower left panel indicates protein expression of total Trx1, determined using anti-hTrx1 Ab (clone 4H9), which detects both mouse Trx 1 and hTrx1. (d) The disulfide oxidoreductase activity of Trx was determined by the insulin reduction assay. Time-dependent reduction of NADPH, determined by spectrophotometry, is shown. ${ }^{*} P<0.01$ compared with NTg. ${ }^{\#} P<0.05$, $\# P<0.01$ compared with 0 min.

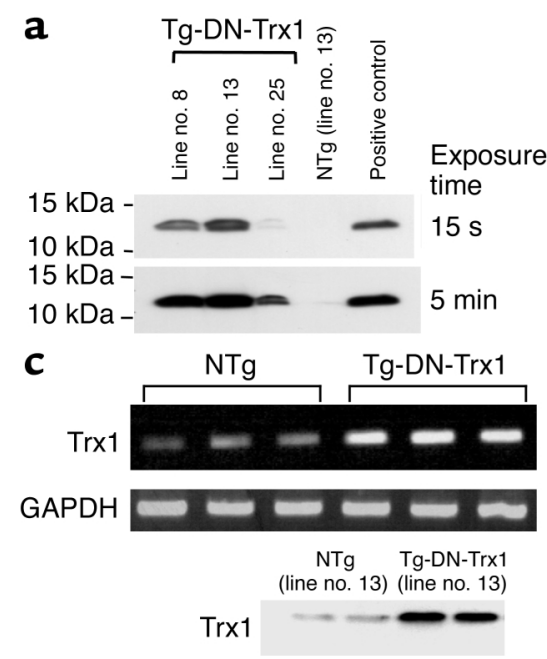

b
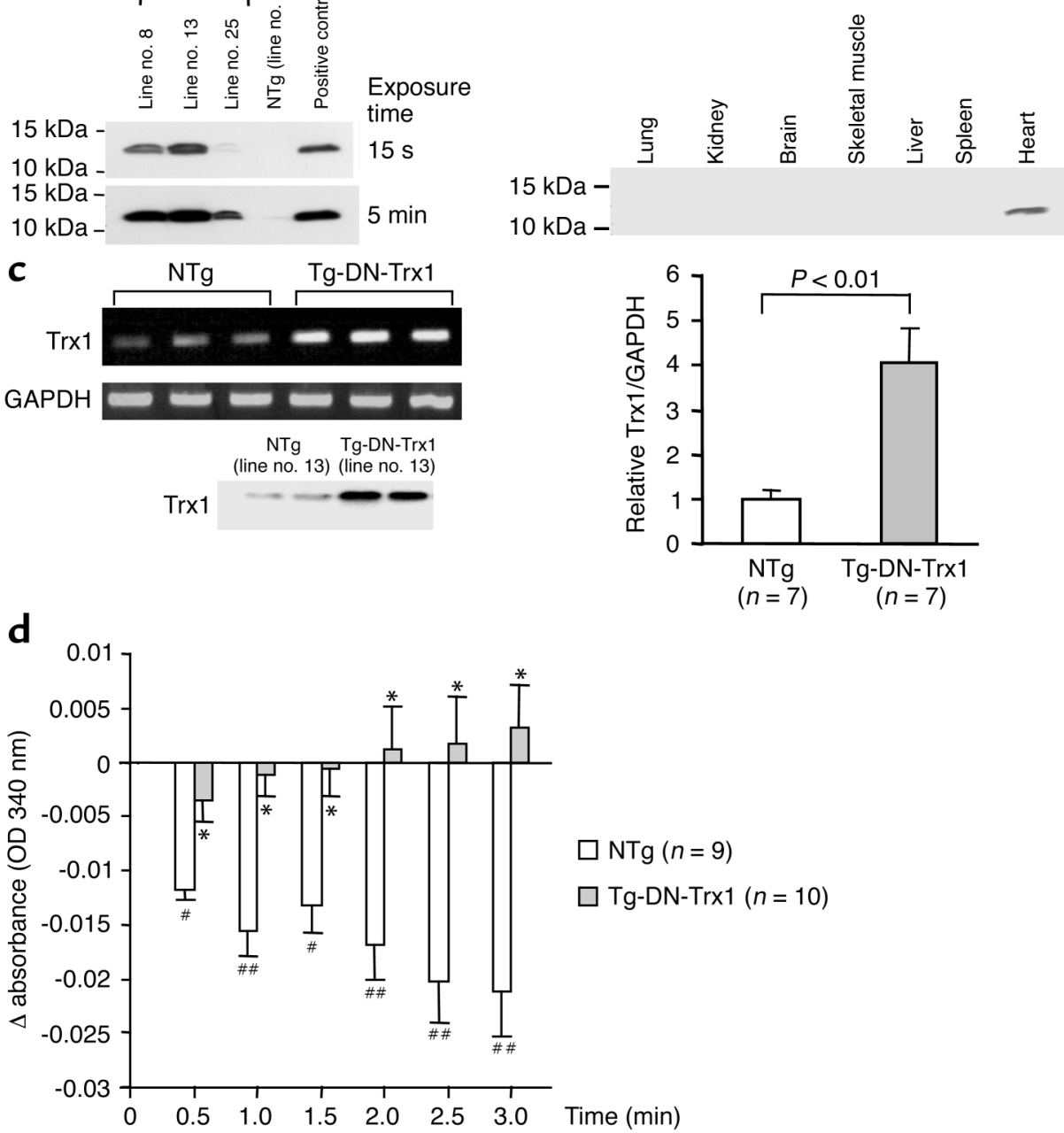

Myocyte cross-sectional area was measured from images captured from silver-stained $1-\mu \mathrm{m}$-thick methacrylate sections as previously described (13). The outline of 100-200 myocytes was traced in each section.

Antioxidant treatment. Age-matched Tg-DN-Trx1 and nontransgenic (NTg) mice were randomized to 4 weeks of treatment with either $\mathrm{N}$-2-mercaptopropionyl glycine (MPG; $100 \mathrm{mg} / \mathrm{kg} / \mathrm{d}$ by intraperitoneal injection) (16) or vehicle (PBS).

Aortic banding. The method to impose pressure overload in mice has been described (13). Mice were anesthetized with a mixture of ketamine $(0.065 \mathrm{mg} / \mathrm{g})$, xylazine $(0.013 \mathrm{mg} / \mathrm{g})$, and acepromazine $(0.002 \mathrm{mg} / \mathrm{g})$ and mechanically ventilated. The left chest was opened at the second intercostal space. Aortic constriction was performed by ligation of the transverse thoracic aorta between the innominate artery and left common carotid artery with a 28 -gauge needle using a 7-0 braided polyester suture. Sham operation was performed without constricting the aorta. To measure arterial pressure gradients, high-fidelity micromanometer catheters (1.4 French; Millar Instruments Inc., Houston, Texas, USA) were used as previously described (13).
Measurement of Ras activation. The heart homogenates were prepared in lysis buffer containing $125 \mathrm{mM}$ HEPES ( $\mathrm{pH} 7.5$ ), $750 \mathrm{mM} \mathrm{NaCl}, 5 \% \mathrm{NP}-40,50 \mathrm{mM} \mathrm{MgCl}_{2}, 5 \mathrm{mM}$ EDTA, $25 \mathrm{mM} \mathrm{NaF}, 1 \mathrm{mM} \mathrm{Na} \mathrm{VO}_{4}, 10 \%$ glycerol, 10 $\mu \mathrm{g} / \mathrm{ml}$ aprotinin, and $10 \mu \mathrm{g} / \mathrm{ml}$ leupeptin. The GTPbound form of Ras was determined using the Ras Activation Assay Kit (Upstate Biotechnology Inc.), which uses binding of the GTP-bound form of Ras to the Rasbinding domain in Raf-1 conjugated with agarose, and subsequent immunoblotting with anti-Ras Ab.

S-thiolation assays. COS-7 cells were transfected with either empty vector (pcDNA3.1) or mammalian expression vectors containing DN-hTrx 1 , antisense rat Trx 1 , or wild-type hTrx1 ( $2 \mu \mathrm{g}$ in 60-mm dishes). Rat Trx1 was cloned by RT-PCR using total RNA prepared from neonatal rat hearts. The antisense construct was made by subcloning rat $\operatorname{Tr} x 1$ into pcDNA3.1 in reverse orientation. Forty-eight hours after transfection, cells were incubated with culture medium containing 0.5 $\mathrm{mM}$ biotinylated cysteine (SynPep Corp., Dublin, California, USA) for 10 minutes. Cells were lysed with $1 \mathrm{ml}$ lysis buffer containing $25 \mathrm{mM}$ HEPES ( $\mathrm{pH} 7.5$ ), 150 $\mathrm{mM} \mathrm{NaCl}, 1 \% \mathrm{NP}-40,10 \mathrm{mM} \mathrm{MgCl}_{2}, 1$ mM EDTA, $10 \%$ 
glycerol, $25 \mathrm{mM} \mathrm{NaF}, 1 \mathrm{mM} \mathrm{Na}_{3} \mathrm{VO}_{4}, 10 \mu \mathrm{g} / \mathrm{ml}$ aprotinin, and $10 \mu \mathrm{g} / \mathrm{ml}$ leupeptin, and the samples were incubated with $40 \mu \mathrm{l}$ slurry of streptavidin-agarose at $4^{\circ} \mathrm{C}$ for 1 hour. After three washes with $1 \mathrm{ml}$ of the lysis buffer, the samples were eluted with Laemmli buffer at $95^{\circ} \mathrm{C}$ for 5 minutes and then subjected to SDS-PAGE and immunoblot analyses with anti-Ras Ab.

Evaluation of cardiac myocyte hypertrophy in vitro. Primary cultures of neonatal rat cardiac myocytes from 1-dayold Crl:(WI)BR-Wistar rats (Charles River Laboratories, Wilmington, Massachusetts, USA) were prepared as previously described (17). Total myocyte protein content was determined as previously described (18). A plasmid containing a 638-bp fragment of the rat ANF promoter linked to firefly luciferase was used to determine the transcriptional activity of the ANF gene as previously described (17). An SV40- $\beta$-galactosidase construct was cotransfected to determine the transfection efficiency.

Morpholino oligo. The sequence of Morpholino antisense oligo designed for rat Trx1 was 5'-GGCAGAACCCGATGGAAATGGAT-3', while that of control Morpholino antisense oligo was $5^{\prime}$-СCTCTTACCTCAGTTACAATTTATA-3'. Morpholino antisense oligo $(2 \mu \mathrm{M})$ was applied to cardiac myocytes grown in either 12- or 24-well plates using the osmotic-loading method according to the manufacturer's instructions (GeneTools LLC, Philomath, Oregon, USA). Twelve hours after initial application, the culture medium was replaced with serum-free cardiac myocyte culture medium without Morpholino antisense oligo, and myocytes were cultured for an additional 36 hours.

Statistics. All values are expressed as mean \pm SEM. Statistical analyses were performed using ANOVA (StatView; SAS Institute Inc., Cary, North Carolina, USA), and, when $F$ values were significant at a $95 \%$ confidence limit, differences among group means were evaluated using the Fisher projected least significant difference post-test procedure for group data, with $P<0.05$ considered significant.

\section{Results}

Generation of $T g$-DN-Trx 1 mice. In order to examine the function of endogenous Trx 1 in the mouse heart in vivo, transgenic mice with cardiac-specific overexpression of dominant negative hTrx1 (Tg-DN-Trx1 mice) were generated using the $\alpha$-myosin heavy chain promoter. We identified five transgenic founders by Southern blot analysis and established four independent lines. Although anti-hTrx1 Ab (2G11) cross-reacts less efficiently with mouse Trx1, endogenous mouse Trx1 was detected by anti-hTrx1 Ab after a long exposure of the blot in NTg mice. DN-hTrx1 is overexpressed in the heart in all lines of Tg-DN-Trx1 mice (Figure 1a and data not shown). The relative levels of DN-hTrx1 expression in the different lines were line no. $13>8>25>24$. In this study, we predominantly characterized line no. 13, unless otherwise stated. We confirmed that DN-hTrx1 is selectively overexpressed in the heart (Figure 1b). The result of RT-PCR indicated that the level of DN-hTrx1 expression was 4.1-fold higher than that of endogenous mouse Trx1 in line no. 13 (Figure 1c). Protein expression of DN-hTrx 1 in line no. 13 was 3.5 -fold higher than that of endogenous Trx1, as determined using another anti$\operatorname{Trx} 1 \mathrm{Ab}(4 \mathrm{H} 9)$ that cross-reacts with both human and rat Trx1. DN-hTrx1 sequestrates NADPH and Trx reductase, essential components of the Trx system, thereby inhibiting disulfide oxidoreductase activity of endogenous Trx1. To test this notion, insulin reduction assays were performed. Although the Trx activity determined by consumption of NADPH was readily detected in NTg mice, it was significantly reduced in Tg-DN-Trx1 mice (Figure 1d), suggesting that the activity of endogenous $\operatorname{Tr} x 1$ is reduced in Tg-DN-Trx1 mice.

Myocardial tissue levels of oxidative stress are elevated in Tg-DN-Trx 1 mice. Myocardial tissue levels of MDA and MDA plus 4-HAE, sensitive indicators of lipid perox-
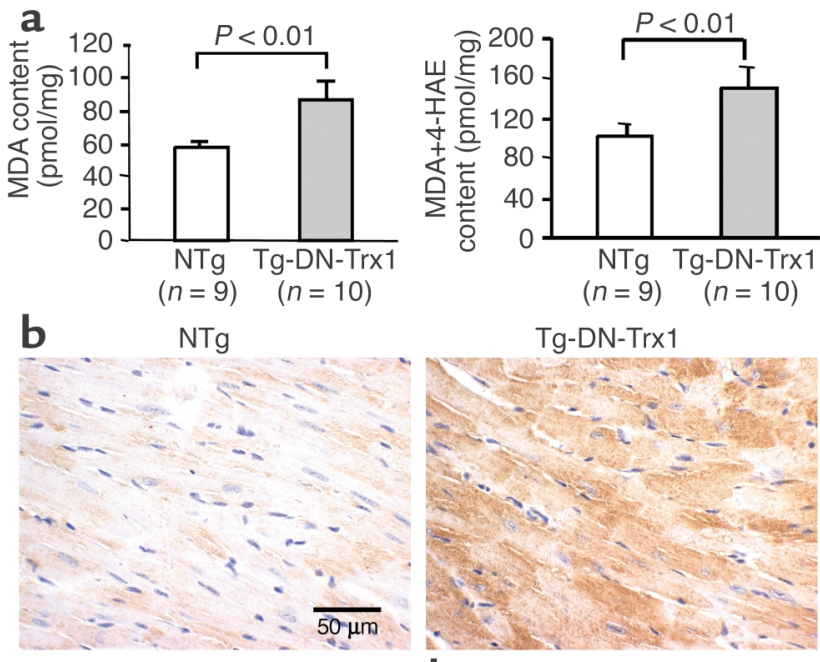

C

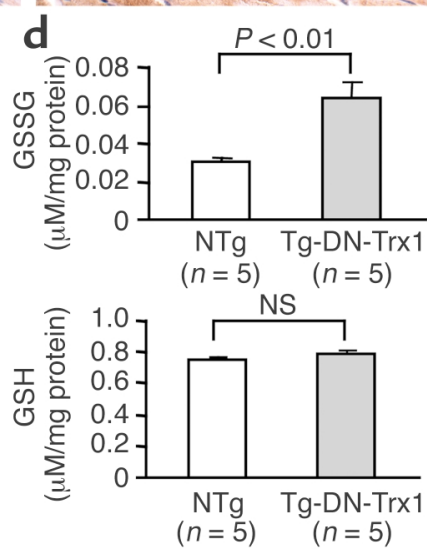

Figure 2

(a) Heart homogenates were prepared from Tg-DN-Trx1 mice and NTg littermates. Tissue levels of MDA alone and MDA plus 4-HAE, markers of lipid peroxidation, were found to be increased in $\operatorname{Tg}-\mathrm{DN}-\mathrm{Trx} 1$ mice. (b) LV myocardial sections were subjected to immunostaining with 8-OHdG, a marker of oxidative DNA damage, which was increased in Tg-DN-Trx1 mice. The result is representative of three experiments. (c) Heart homogenates were prepared from Tg-DN-Trx1 mice and NTg littermates. Immunoblot analyses of MnSOD, CuZnSOD, and catalase are shown. Note that neither the level of MnSOD nor the level of catalase differed between Tg-DN-Trx1 and NTg mice, while that of CuZnSOD was higher (about 2.5-fold) in Tg-DN-Trx1 mice. $n=3$. (d) Tissue levels of GSSG and GSH were determined using fresh samples. 
a

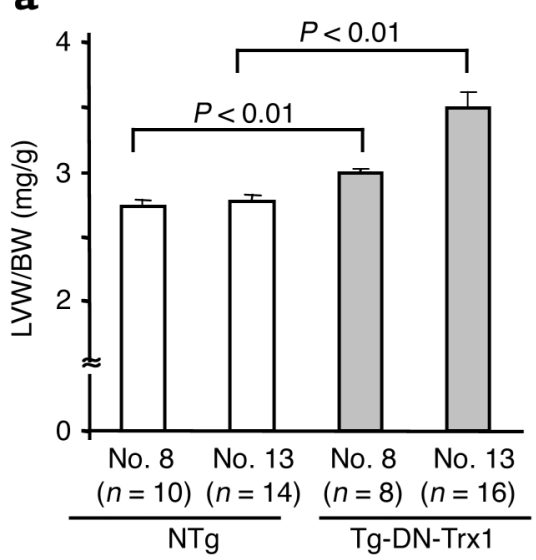

C

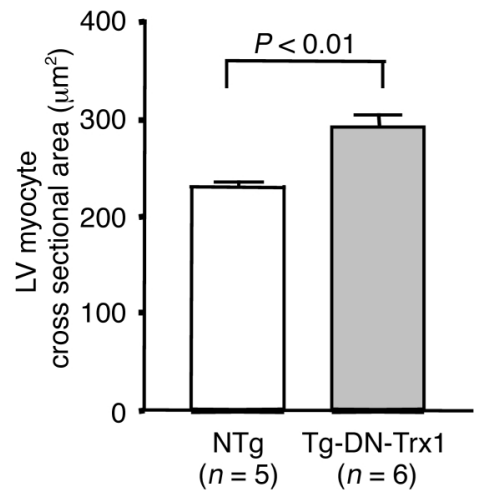

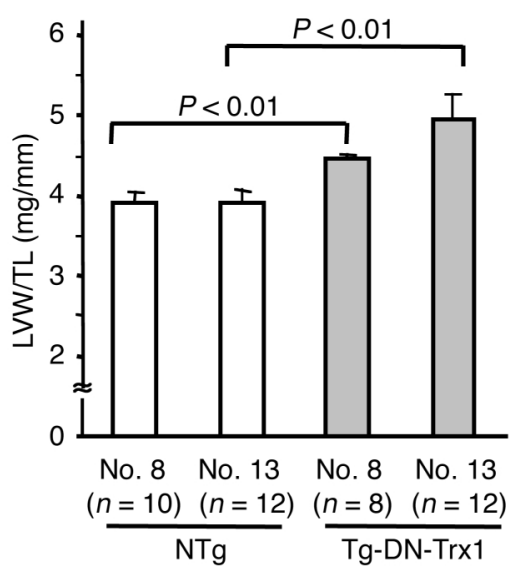

d

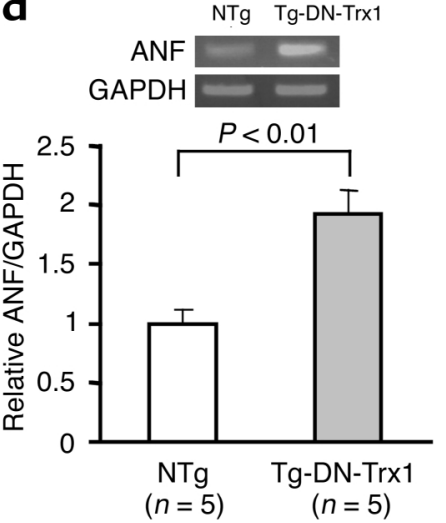

b

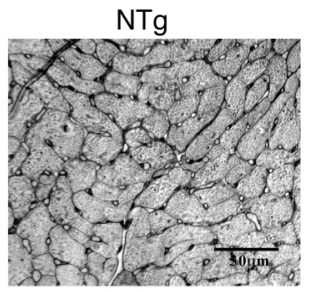

Tg-DN-Trx1

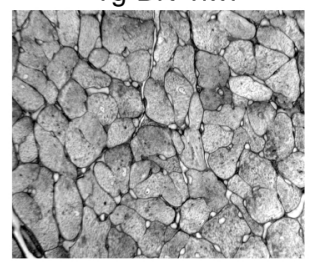

e
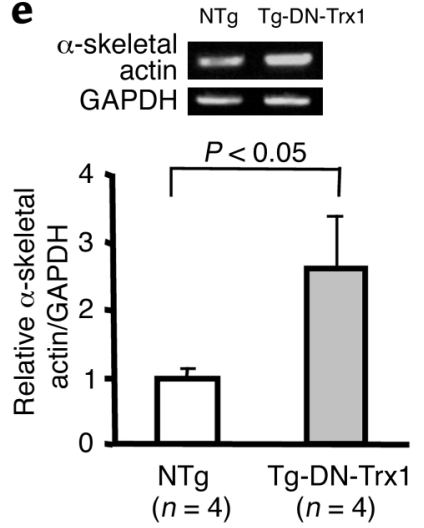

Figure 3

(a) LVW/BW (mg/g; left) and LVW/TL (mg/mm; right) were determined in Tg-DN-Trx1 mice (line nos. 8 and 13) and NTg littermates. The age of the animals was $85 \pm 3$ days in Tg-DN-Trx1 line no. 8, $81 \pm 4$ days in Tg-DN-Trx1 line no. 13, $84 \pm 4$ days in NTg line no. 8 , and $79 \pm 5$ days in NTg line no. 13. (b) Silver staining of LV myocardial sections, showing LV myocyte cross-sectional area in Tg-DN-Trx 1 mice and NTg littermates. (c) Mean LV myocyte cross-sectional area was determined from 100-200 myocytes in each animal. The experiments were conducted using five NTg and six Tg-DN-Trx1 mice. (d and e) mRNA expression of ANF (d), $\alpha$-skeletal actin (e), and GAPDH was determined by RT-PCR. The intensity of the ethidium bromide bands was quantitated. The intensity of ANF or $\alpha$-skeletal actin bands was normalized by that of GAPDH bands.

idation, were significantly elevated in Tg-DN-Trx1 mice compared with those in NTg littermates (Figure 2a). Myocardial levels of $8-\mathrm{OHdG}$, a sensitive indicator of oxidative DNA damage, were also elevated in Tg-DN-Trx1 mice (Figure $2 \mathrm{~b}$ ). These results suggest that the tissue levels of oxidative stress are significantly elevated in Tg-DN-Trx1 mice.

Other antioxidant mechanisms are not downregulated in $T g-D N-\operatorname{Tr} x 1$ mice. We examined whether overexpression of DN-hTrx1 affects other antioxidant mechanisms in the heart. Immunoblot analyses indicated that expression of MnSOD and catalase was not significantly different between Tg-DN-Trx1 mice and NTg littermates (Figure 2c). By contrast, expression of CuZnSOD was slightly higher (twofold) in Tg-DN-Trx1 mice (Figure 2c). Although the myocardial level of GSSG, an oxidized form, was significantly elevated in Tg-DN-Trx1 mice, the amount of GSH was not significantly different between Tg-DN-Trx1 and NTg mice (Figure 2d).

$\operatorname{Tg}$-DN-Trx 1 mice show base-line cardiac bypertrophy. At 3 months, both the LV weight/body weight ratio
(LVW/BW) and the LV weight/tibial length ratio (LVW/TL) were significantly greater in Tg-DN-Trx1 mice (line no. 13) than in NTg littermates (Figure 3a). Smaller but significant hypertrophy was also observed in the other three lines (nos. 8, 24, and 25) (Figure 3a and data not shown). LV cardiac myocyte cross-sectional area was significantly greater in Tg-DN-Trx1 mice than in NTg littermates (Figure 3, b and c). The results of RT-PCR analyses indicated that mRNA expression of ANF and $\alpha$-skeletal actin, the fetal-type genes, was significantly elevated in Tg-DN-Trx 1 mice (Figure 3, $\mathrm{d}$ and e). H\&E staining of the LV showed that there was no significant infiltration of inflammatory cells in Tg-DNTrx1 mice. Picric acid Sirius red staining indicated that there was no significant interstitial fibrosis in Tg-DNTrx1 mice (data not shown).

Echocardiographic studies showed that LV septum and posterior wall thickness in both diastolic and systolic phases was significantly greater in Tg-DN-Trx1 than in NTg mice $(P<0.01$; Table 1$)$. LVEDD, LVESD, $\mathrm{LVEF}$, and \%FS were all maintained in Tg-DN-Trx1 mice. 


\section{Table 1}

Base-line echocardiographic data of Tg-DN-Trx1 mice and NTg littermates at the age of 3 months

\begin{tabular}{lcc}
\hline & NTg $(n=12)$ & Tg-DN-Trx $1(n=15)$ \\
DSEP Wt $(\mathrm{mm})$ & $0.715 \pm 0.049$ & $0.883 \pm 0.041^{\mathrm{A}}$ \\
LVEDD $(\mathrm{mm})$ & $3.837 \pm 0.160$ & $3.834 \pm 0.159$ \\
DP Wt $(\mathrm{mm})$ & $0.673 \pm 0.043$ & $0.844 \pm 0.065^{\mathrm{A}}$ \\
SSEP Wt $(\mathrm{mm})$ & $1.117 \pm 0.067$ & $1.254 \pm 0.082^{\mathrm{A}}$ \\
LVESD $(\mathrm{mm})$ & $2.423 \pm 0.085$ & $2.439 \pm 0.093$ \\
SP Wt $(\mathrm{mm})$ & $0.981 \pm 0.092$ & $1.112 \pm 0.087^{\mathrm{A}}$ \\
EF & $0.751 \pm 0.026$ & $0.740 \pm 0.029$ \\
\%FS & $37.2 \pm 2.2$ & $35.9 \pm 2.4$ \\
HR (beats per min) & $402 \pm 29$ & $392 \pm 41$
\end{tabular}

Data are mean \pm SEM. ${ }^{A} P<0.01$ compared with NTg mice. DSEP Wt , diastolic septal wall thickness; DP Wt, diastolic posterior wall thickness; SSEP Wt, systolic septal wall thickness; SP Wt, systolic posterior wall thickness; EF, LV ejection fraction; $H R$, heart rate.

These results suggest that Tg-DN-Trx1 mice exhibit cardiac hypertrophy with well-maintained LV function.

Base-line cardiac hypertrophy in Tg-DN-Trx 1 mice is mediated by increased levels of oxidative stress. In order to characterize the involvement of oxidative stress in base-line cardiac hypertrophy of Tg-DN-Trx1 mice, we treated mice with MPG, a well-established antioxidant (16), for 4 weeks starting at the age of 1 month. MPG treatment did not affect body weight or heart weight significantly in control mice. By contrast, MPG significantly reduced heart weight in $\mathrm{Tg}-\mathrm{DN}$ -

Trx1 mice compared with saline injection. As a result, MPG normalized both LVW/BW and LVW/TL in Tg-DN-Trx1 mice to the levels found in control mice (Figure 4, $a$ and $b$ ). In addition, we confirmed that the myocardial level of $8-\mathrm{OHdG}$ was reduced by MPG treatment in TgDN-Trx1 mice (Figure 4c). These results are consistent with the notion that base-line cardiac hypertrophy in Tg-DN-Trx 1 mice is maintained by increased levels of oxidative stress.

Inbibition of endogenous Trx 1 enhances pressure overload-induced cardiac bypertrophy. In control mouse hearts, transverse aortic banding for 1 or 2 weeks caused upregulation of endogenous Trx1 (Figure 5a). In order to test whether endogenous Trx1 also plays an important role in regulating cardiac

\section{Figure 4}

hypertrophy induced by pathologic stimuli, we applied aortic banding to Tg-DN-Trx1 and NTg mice. After 2 weeks, the pressure gradient was similar between $\mathrm{Tg}$ DN-Trx 1 mice and NTg littermates (105 \pm 8 vs. $103 \pm 4$ $\mathrm{mmHg}$ ). Although the cardiac content of MDA and 4-HAE increased significantly in response to pressure overload in both Tg-DN-Trx1 and NTg mice, the degree of the increases in these indicators of lipid peroxidation was significantly greater in Tg-DN-Trx1 mice (Figure $5 \mathrm{~b})$. Aortic banding caused significant increases in LVW/BW and LVW/TL in both Tg-DN-Trx1 and NTg mice compared with sham operation (Figure $5 \mathrm{c}$ and data not shown). Interestingly, the percentage increase in LVW/BW and LVW/TL was significantly greater in Tg-DN-Trx 1 than in NTg mice (Figure $5 \mathrm{~d}$ and data not shown). Similar results were observed after 4 weeks of aortic banding (Figure 5, e and f). These results suggest that endogenous $\operatorname{Tr} 1$ negatively regulates the extent of cardiac hypertrophy in response to pressure overload.

The results of echocardiographic measurements conducted at 2 weeks indicated that cardiac function was maintained in both Tg-DN-Trx1 and NTg mice (Table 2). The LV systolic function was slightly reduced after 4 weeks in both groups (Table 3). However, dilation of the LV chamber was not observed in either NTg or Tg-DN-Trx1 mice, and LVESD became even smaller in Tg-DN-Trx1 mice after 4 weeks of banding (Table 3). These results suggest that inhibition of endogenous a
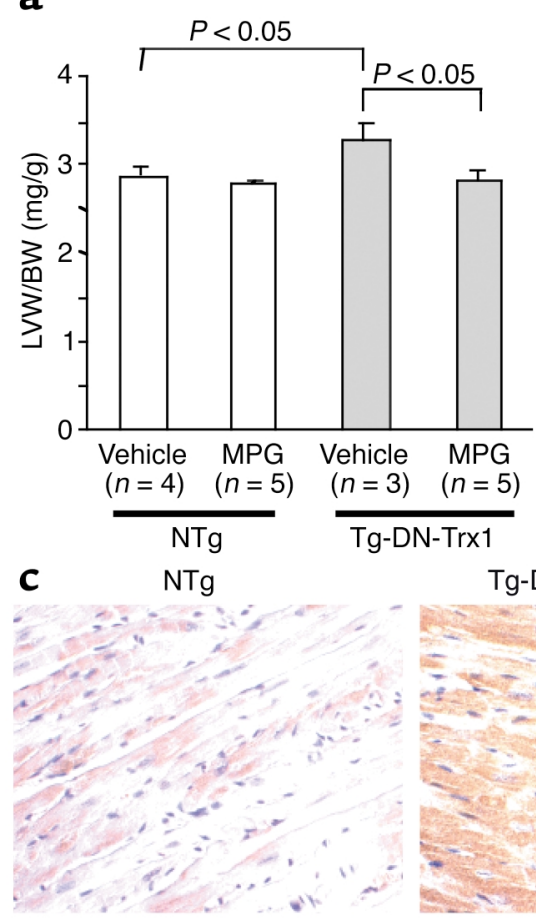

Tg-DN-Trx1 + vehicle

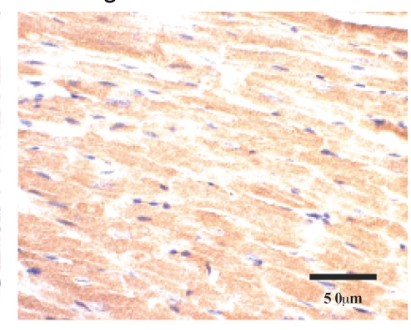

b

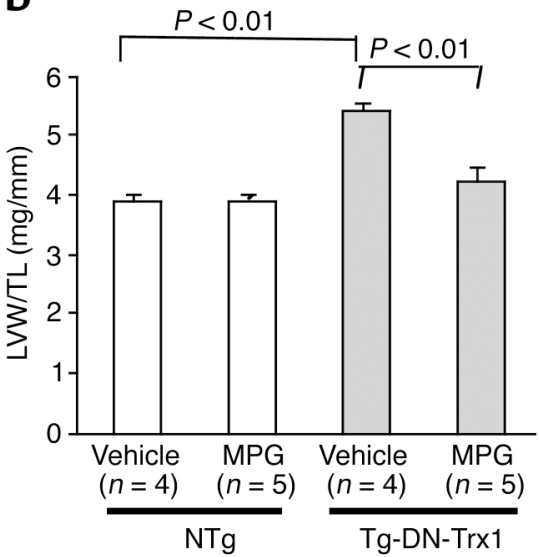

The effect of antioxidant treatment on base-line cardiac hypertrophy in Tg-DN-Trx1 mice. Tg-DN-Trx1 mice and NTg littermates were treated with an intraperitoneal injection of MPG $(100 \mathrm{mg} / \mathrm{kg} / \mathrm{d})$ or saline (vehicle) for 4 weeks. LWW/BW (a) and LWW/TL (b) were determined. (c) LV myocardial sections obtained from NTg mice (left) and Tg-DN-Trx1 mice treated with saline (center) or MPG (right) were subjected to immunostaining with $8-\mathrm{OHdG}$. 

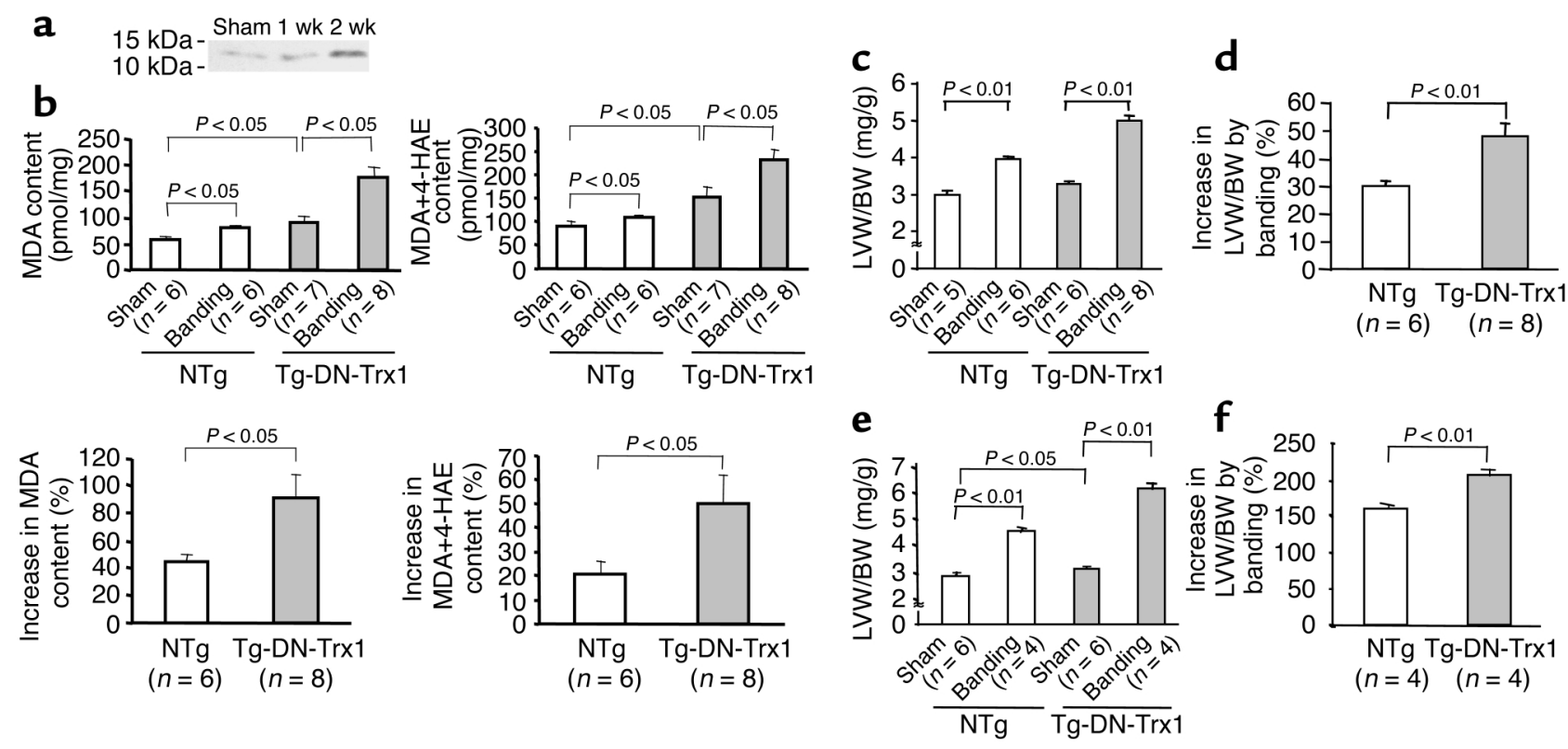

\section{Figure 5}

Tg-DN-Trx1 mice and NTg littermates were subjected to either transverse aortic banding or sham operation. (a) The effect of pressure overload on expression of endogenous mouse Trx 1 in NTg mice. The result is representative of four experiments. (b-d) Aortic banding was applied for 2 weeks. (b) The effect of pressure overload on oxidative stress in the heart. The heart content of MDA and of MDA plus 4-HAE, the percentage increase in MDA, and the percentage increase in MDA plus 4-HAE are shown. (c and d) LVW/BW (c) and percentage increase in $\mathrm{LWW} / \mathrm{BW}$ in response to aortic banding $(\mathbf{d})$ were determined. Similar results were obtained for LWW/TL. The percentage increase in LWW/BW and LWW/TL (not shown) was significantly greater in Tg-DN-Trx1 than in NTg mice in response to pressure overload. (e and $\mathbf{f}$ ) Aortic banding was applied for 4 weeks. LWW/BW (e) and percentage increase in LW/BW in response to aortic banding (f) were determined. The percentage increase in LWW/BW was significantly greater in Tg-DN-Trx1 than in NTg mice.

Trx stimulates a concentric form of cardiac hypertrophy in response to pressure overload.

Activity of the Ras-Raf-1-ERK pathway, rather than the JNK or p38-MAPK pathway, is enhanced in the hearts of Tg-DNTrx 1 mice. In order to examine the mechanism of cardiac hypertrophy in $\mathrm{Tg}$-DN-Trx1 mice, we examined whether members of the MAPK family are activated in Tg-DNTrx1 hearts. Interestingly, activity of extracellular signal-regulated kinase (ERK) and that of its upstream regulators, Raf- 1 and Ras, were significantly elevated in Tg-DN-Trx1 compared with NTg hearts (Figure 6, a-c). ERK activation in Tg-DN-Trx1 mice was further enhanced by aortic banding (Figure 6a, lower panel). It has been shown that oxidation of the cysteine residues (S-thiolation) activates Ras (19). To examine whether Ras undergoes S-thiolation in the presence of DNhTrx1, S-thiolation assays were conducted using COS-7 cells. S-thiolation of Ras was observed in the presence of DN-hTrx1, but not either empty vector or wild-type Trx1. S-thiolation of Ras was also observed in the presence of antisense $\operatorname{Tr} x 1$, suggesting that decreases in the activity of Trx1 cause S-thiolation of Ras (Figure 6d). Although it has been shown that DN-hTrx1 activates ASK1 (20), an upstream kinase of p38-MAPK and JNKs, activities of JNKs and p38-MAPK were not significantly elevated in Tg-DN-Trx1 mice (Figure 6, e and f).

Transgenic mice with cardiac-specific overexpression of Trx 1 do not develop cardiac hypertrophy. Cardiac-specific overexpression of DN-hTrx1 may stimulate hypertrophy through redox-independent protein-protein interactions (4). In addition, wild-type Trx 1 works as a growth factor in some cell types $(21,22)$. In order to test whether cardiac hypertrophy seen in Tg-DN-Trx1 mice is mediated by redox-independent effects of Trx1, we generated transgenic mice with cardiac-specific overexpression of wild-type Trx1 (Tg-Trx1 mice). We generated five transgenic lines and confirmed that wild-type Trx1 is overexpressed in a heart-specific manner (Figures 7 , $a$ and $b$ ). We predominantly characterized line no. 18, where expression of wild-type Trx 1 in the heart, determined by RT-PCR, was 4.5 -fold higher than in NTg mice (Figure 7c). Protein expression of wild-type Trx 1 in line no. 18, determined using anti-Trx1 Ab (4H9), was 3.4-fold higher than that of endogenous Trx1. We confirmed that the Trx activity is significantly elevated in $\operatorname{Tg}-\operatorname{Tr} x 1$ hearts, which contrasted markedly with the Trx activity in Tg-DN-Trx1 hearts (Figure 7d). At 3 months, neither LVW/BW nor LVW/TL in Tg-Trx1 mice was different from that in NTg mice (Figure 7e). Similar results were confirmed in all other Tg-Trx1 lines generated (data not shown). Echocardiographic measurements indicated that chamber sizes and cardiac function in $\operatorname{Tg}-\operatorname{Tr} 1$ mice were normal, and wall thickness in Tg-Trx1 did not differ from that in NTg mice (Table 4). If the prohypertrophic effect of DN-hTrx1 is mediated by decreases in endogenous Trx 1 activity, overexpression of Trx1 should exhibit antihypertrophic effects. To test this hypothesis, aortic banding was conducted for 
Table 2

Echocardiographic data of Tg-DN-Trx1 mice and NTg littermates before and after aortic banding for 2 weeks

\begin{tabular}{|c|c|c|c|c|}
\hline & \multicolumn{2}{|c|}{ NTg $(n=4)$} & \multicolumn{2}{|c|}{$\operatorname{Tg}-\mathrm{DN}-\operatorname{Trx} 1(n=7)$} \\
\hline & Before banding & After banding & Before banding & After banding \\
\hline DSEP Wt $(\mathrm{mm})$ & $0.613 \pm 0.037$ & $0.829 \pm 0.035^{\mathrm{A}}$ & $0.733 \pm 0.019^{B}$ & $1.137 \pm 0.021^{\mathrm{A}, \mathrm{C}}$ \\
\hline LVEDD (mm) & $3.970 \pm 0.188$ & $3.796 \pm 0.078$ & $3.697 \pm 0.135$ & $3.521 \pm 0.122$ \\
\hline $\mathrm{DPWt}(\mathrm{mm})$ & $0.568 \pm 0.042$ & $0.829 \pm 0.045^{A}$ & $0.747 \pm 0.068^{C}$ & $1.128 \pm 0.024^{\mathrm{A}, \mathrm{C}}$ \\
\hline SSEP Wt $(\mathrm{mm})$ & $1.005 \pm 0.070$ & $1.158 \pm 0.048$ & $1.124 \pm 0.033^{B}$ & $1.461 \pm 0.016^{\mathrm{A}, \mathrm{C}}$ \\
\hline LVESD (mm) & $2.615 \pm 0.148$ & $2.542 \pm 0.075$ & $2.321 \pm 0.101$ & $2.298 \pm 0.105$ \\
\hline SP Wt (mm) & $0.843 \pm 0.069$ & $1.199 \pm 0.066^{\mathrm{A}}$ & $1.023 \pm 0.053^{B}$ & $1.466 \pm 0.018^{\mathrm{A}, \mathrm{C}}$ \\
\hline $\mathrm{EF}$ & $0.715 \pm 0.012$ & $0.699 \pm 0.015$ & $0.753 \pm 0.018$ & $0.723 \pm 0.011$ \\
\hline$\% F S$ & $34.3 \pm 0.9$ & $33.1 \pm 1.2$ & $37.3 \pm 1.5$ & $34.9 \pm 0.9$ \\
\hline HR & $430 \pm 44$ & $452 \pm 11$ & $408 \pm 21$ & $463 \pm 30$ \\
\hline BW (g) & $25.50 \pm 1.32$ & $27.00 \pm 1.35$ & $25.714 \pm 1.46$ & $25.86 \pm 0.99$ \\
\hline Age (d) & $78.8 \pm 0.5$ & $92.8 \pm 0.5$ & $83.4 \pm 2.4$ & $97.4 \pm 2.4$ \\
\hline
\end{tabular}

Data are mean \pm SEM. ${ }^{A} P<0.01$ compared with base line. ${ }^{B} P<0.05,{ }^{C} P<0.01$ compared with NTg mice.

2 weeks using Tg-Trx1 and NTg mice, and the pressure gradient was similar between the two groups. As expected, increases in oxidative stress (MDA alone and MDA plus 4-HAE) and in LV hypertrophy (LVW/BW), induced by pressure overload, were smaller in Tg-Trx1 than in NTg mice (Figure 7, f and g).

Antisense inbibition of Trx 1 causes bypertrophy in cultured cardiac myocytes. In order to confirm that the hypertrophic effect of DN-hTrx 1 is mediated by decreases in the endogenous Trx1 activity, we examined the effect of antisense inhibition of Trx1. We confirmed that antisense inhibition by either Morpholino oligo or plasmid significantly decreased expression of endogenous Trx 1 in cardiac myocytes and COS-7 cells (Figure 8 , a and d). Morpholino antisense Trx1 oligo treatment significantly increased the total protein content (Figure $8 \mathrm{~b}$ ) as well as MDA and MDA plus 4-HAE content (Figure 8c) in cardiac myocytes compared with control Morpholino oligo treatment. Cotransfection assays indicated that both DN-hTrx1 and antisense Trx1 significantly increased the transcriptional activity of ANF, a cardiac-hypertrophy marker gene, while empty vector and wild-type Trx1 did not (Figure 8e). These results suggest that antisense inhibition of Trx 1 exhibits hypertrophic effects similar to those of DN-hTrx 1 in cultured cardiac myocytes.

\section{Discussion}

Although it has been suggested that one of the most important functions of $\operatorname{Trx} 1$ is as an antioxidant (4), the importance of $\operatorname{Trx} 1$ among cellular antioxidant mechanisms has not been demonstrated clearly in any organs in vivo. Our results suggest that cardiac-specific overexpression of redox-inactive $\operatorname{Tr} x 1$ in transgenic mice reduced disulfide oxidoreductase activity of endogenous Trx in the heart. In this animal model, oxidative stress in the heart was significantly increased under both basal and pathologic conditions. This clearly indicates that Trx 1 plays an important role as a regulator of oxidative stress in the heart in vivo.

The cellular environment is maintained in reducing conditions, and therefore intracellular proteins contain free thiol, rather than disulfides, under physiological conditions (2). Trx1 reduces intracellular proteins with disulfides through its disulfide oxidoreductase activity (4). Oxidative stress in the heart, in various cardiovas-

\section{Table 3}

Echocardiographic data of Tg-DN-Trx1 mice and NTg littermates before and after aortic banding for 4 weeks

\begin{tabular}{|c|c|c|c|c|}
\hline & \multicolumn{2}{|c|}{$\operatorname{NTg}(n=4)$} & \multicolumn{2}{|c|}{$\operatorname{Tg}-\mathrm{DN}-\operatorname{Tr} x 1(n=4)$} \\
\hline & Before banding & After banding & Before banding & After banding \\
\hline DSEP Wt $(\mathrm{mm})$ & $0.724 \pm 0.037$ & $1.111 \pm 0.054^{\mathrm{A}}$ & $0.858 \pm 0.051^{\mathrm{B}}$ & $1.419 \pm 0.061^{\mathrm{A}, \mathrm{C}}$ \\
\hline LVEDD $(\mathrm{mm})$ & $4.055 \pm 0.079$ & $3.937 \pm 0.035$ & $3.939 \pm 0.118$ & $3.475 \pm 0.056^{\mathrm{A}, \mathrm{B}}$ \\
\hline $\mathrm{DPWt}(\mathrm{mm})$ & $0.684 \pm 0.038$ & $0.979 \pm 0.025^{\mathrm{A}}$ & $0.798 \pm 0.036^{\mathrm{B}}$ & $1.295 \pm 0.006^{\mathrm{A}, \mathrm{C}}$ \\
\hline SSEP Wt $(\mathrm{mm})$ & $1.139 \pm 0.067$ & $1.519 \pm 0.041^{\mathrm{A}}$ & $1.186 \pm 0.054$ & $1.761 \pm 0.080^{\mathrm{A}, \mathrm{B}}$ \\
\hline $\operatorname{LVESD}(\mathrm{mm})$ & $2.632 \pm 0.024$ & $2.737 \pm 0.058$ & $2.551 \pm 0.103$ & $2.426 \pm 0.050^{C}$ \\
\hline SP Wt $(\mathrm{mm})$ & $1.061 \pm 0.037$ & $1.376 \pm 0.044^{\mathrm{A}}$ & $1.117 \pm 0.069^{B}$ & $1.573 \pm 0.059^{\mathrm{A}, \mathrm{B}}$ \\
\hline $\mathrm{EF}$ & $0.724 \pm 0.013$ & $0.663 \pm 0.014^{\mathrm{A}}$ & $0.728 \pm 0.015$ & $0.659 \pm 0.015^{\mathrm{A}}$ \\
\hline \%FS & $35.0 \pm 1.1$ & $30.5 \pm 1.0^{\mathrm{A}}$ & $35.2 \pm 1.2$ & $30.2 \pm 1.0^{\mathrm{A}}$ \\
\hline $\mathrm{HR}$ & $425 \pm 25$ & $436 \pm 42$ & $406 \pm 21$ & $446 \pm 42$ \\
\hline BW $(g)$ & $29.25 \pm 2.87$ & $28.00 \pm 1.29$ & $27.00 \pm 1.47$ & $28.25 \pm 0.48$ \\
\hline Age (d) & $134.5 \pm 0.9$ & $158.5 \pm 0.9$ & $129.3 \pm 0.8$ & $159.3 \pm 0.8$ \\
\hline
\end{tabular}

Data are mean $\pm \mathrm{SEM}$. ${ }^{A} P<0.05$ compared with base line. ${ }^{\mathrm{B} P}<0.05,{ }^{C} P<0.01$ compared with $\mathrm{NTg}$ mice. 


\section{Figure 6}

The activities of the Ras-Raf-1-ERK pathway, p38-MAPK, and p46/p54-JNK1 were examined in Tg-DN-Trx1 mice and NTg littermates. (a) Some mice were subjected to aortic banding for 2 weeks. Activities of p42/p44-ERK (a), Raf-1 (b), p38-MAPK (e), and p46/p54-JNK1 (f) were determined using anti-phosphospecific Ab's. The same filter was reprobed with respective non-phosphospecific Ab. (e) Cell extracts prepared from cardiac myocytes overexpressing mammalian sterile 20-like kinase 1 (Mst1) were used as positive control (P/C). The gel picture is representative of three to ten experiments in each immunoblot analysis. (c) The activity of Ras was determined using the Ras-binding domain in Raf-1 coupled with agarose. (d) S-thiolation of Ras was determined as described in Methods. COS-7 cells grown in 60-mm dishes were transfected with the indicated expression plasmids $(2 \mu \mathrm{g})$. Cells were incubated with biotinylated cysteine $(0.5$ $\mathrm{mM}$ ), and S-thiolated proteins were isolated by streptavidin-Sepharose. Samples were subjected to immunoblot analyses with anti-Ras Ab. AS-Trx1, antisense Trx1.

cular disease conditions, modifies proteins with redox-sensitive cysteine residues by a process called S-thiolation, thereby modulating the function of the protein and intracellular-signaling mechanisms $(4,23)$. We believe that Tg-DN-Trx1 mice should be useful in identification of pathologically relevant S-thiolated proteins controlled by endogenous Trx 1 .

Another important finding in this report is that inhibition of endogenous Trx 1 in the heart induces cardiac hypertrophy under basal conditions and enhances cardiac hypertrophy in response to pressure overload. This observation is intriguing because it has been suggested that $\operatorname{Trx} 1$ can work as a secreted growth factor, thereby stimulating proliferation of cancer cells (21). Recently, it was shown that overexpression of vitamin D3-upregulated protein-1 (VDUP1) prevents PDGF-induced proliferation of VSMCs, presumably by inhibiting the activity of endogenous $\operatorname{Trx} 1$ (22). Although the fact that inhibition of endogenous Trx 1 by VDUP1 prevents cell proliferation in VSMCs suggests that the effect of Trx1 on cell growth differs between cardiac myocytes and smooth muscle cells, it is possible that VDUP1 has additional effects besides inhibition of Trx1. Alternatively, as we discuss below, the antioxidant action of Trx1 may have a stronger influence as an inhibitor of hypertrophic growth of cardiac myocytes than as a growth factor.

Recent evidence suggests that reactive oxygen species play an important role in mediating cardiac hypertrophy $(1,2,16,24-27)$. Application of antioxidant molecules has been shown to prevent cardiac hypertrophy by d pcDNA DN-Trx1 AS-Trx1 Trx1

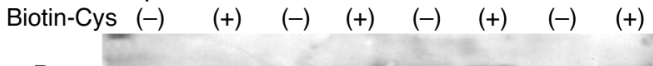

Ras -
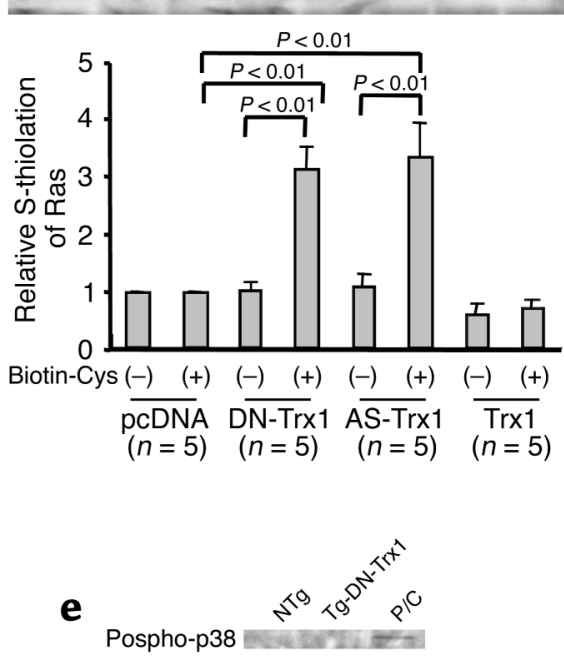

p38

f
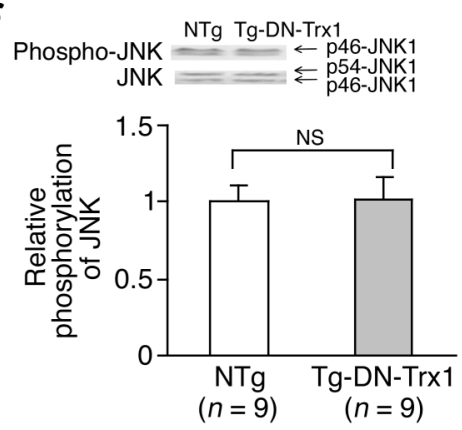

humoral factors in cultured cardiac myocytes as well as by pressure overload in vivo $(16,24-26)$. Inhibition of CuZnSOD, an important cytosolic antioxidant molecule, by diethylthiocarbamic acid induces cardiac hypertrophy through a reactive oxygen species-dependent mechanism in neonatal rat cardiac myocytes (27). However, the effect of inhibition of antioxidant mechanisms on cardiac hypertrophy has not been tested in vivo, since it is difficult to selectively abolish the antioxidant mechanism without toxicity in vivo. Our results extend these previous observations in vitro and provide strong evidence that increased levels of oxidative stress caused by selective inhibition of an antioxidant mechanism stimulate cardiac hypertrophy in vivo.

It has been shown that overexpression of Trx 1 protects the brain from ischemia/reperfusion injury (28), suggesting that Trx1 has cell-protective effects. Overexpression of VDUP1 promotes apoptosis in neonatal rat cardiac myocytes (7). Interestingly, however, inhibition of endogenous Trx1 in transgenic mice neither caused decreases in cardiac function at base line nor facilitated decompensation after aortic banding. In fact, 4 weeks of aortic banding in Tg-DN-Trx1 mice caused decreases in LVESD due to concentric hypertrophy rather than LV 
chamber dilation (13). Thus, our results suggest that, although overexpression of Trx 1 may exhibit cell-protective effects, endogenous $\operatorname{Trx} 1$ primarily regulates hypertrophy rather than cell-protective mechanisms. Alternatively, some aspects of the genetic background of the FVB strain might have contributed to the wellmaintained LV function seen in Tg-DN-Trx1 mice despite higher levels of hypertrophy.

In addition to regulating ROS, Trx1 has multiple cellular functions through interaction with intracellularsignaling molecules, such as ASK1 (29), PKCs (30),
p40Phox (31), and PTEN (32), and nuclear transcription factors, including NF- $\mathrm{KB}(33)$, glucocorticoid receptor (34), and p53 (35). Thus, interaction between Trx1 and downstream molecules may directly regulate cellular function independently of redox regulation. It has recently been shown that Trx1 regulates ASK1 through a redox-independent mechanism, which may contribute to hypertrophic phenotype in Tg-DN-Trx1 mice (20). We believe, however, that downregulation of Trx1 activity and subsequent increases in oxidative stress play a major role in mediating cardiac hypertrophy in Tg-DN-
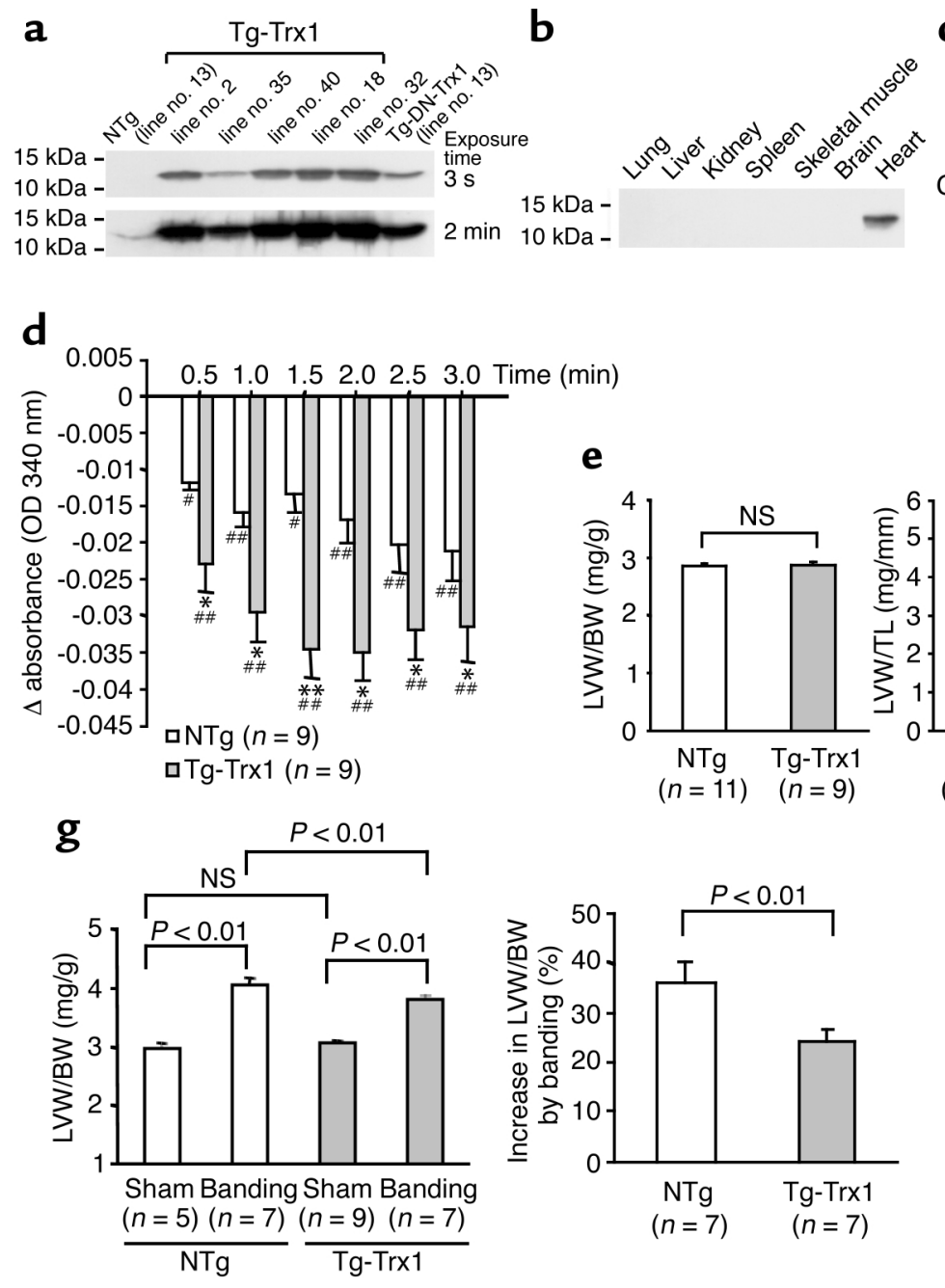

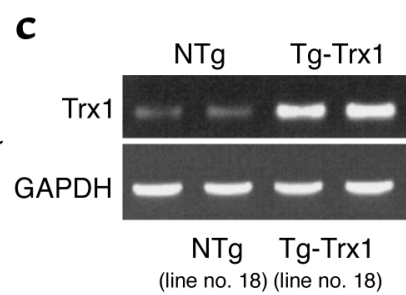

$\operatorname{Trx1}$

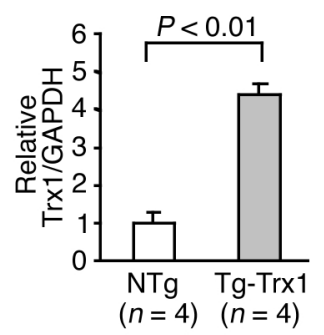

\section{Figure 7}

Characterization of transgenic mice with cardiac-specific overexpression of wild-type $\operatorname{Tr} 1$ ( $\operatorname{Tg}$ - $\operatorname{Tr} x 1$ mice). (a) Heart homogenates were prepared from Tg-Trx1 mice and NTg littermates. Immunoblot analyses were conducted using anti-hTrx1 Ab. Short (3-second) and long (2-minute) exposures of the immunoblot are shown. After long exposure, endogenous mouse Trx 1 was detected. (b) Tissue homogenates were prepared from various organs. Immunoblot analyses were conducted using anti-hTrx1 Ab. (c) RT-PCR analyses of Trx1 and GAPDH. Total RNA was extracted from Tg-Trx1 (line no. 18) and NTg mice. The lower left panel indicates protein expression of total Trx1, determined using anti-hTrx1 Ab (clone 4H9). (d) The disulfide oxidoreductase activity of Trx was determined by the insulin reduction assay. Time-dependent reduction of NADPH, determined by spectrophotometry, is shown. ${ }^{*} P<0.05 ;{ }^{*} P<0.01$ compared with NTg. ${ }^{*} P<0.05$, ${ }^{\# \# P}<0.01$ compared with $0 \mathrm{~min}$. (e) LWW/BW ( $\mathrm{mg} / \mathrm{g}$; left) and LWW/TL ( $\mathrm{mg} / \mathrm{mm}$; right) were determined in Tg-Trx1 mice (line no. 18) and NTg littermates (line no. 18). The age of the animals was $94 \pm 9$ days in Tg-Trx1 and $97 \pm 7$ days in NTg mice. Base-line hypertrophy was not observed in any other lines of Tg-Trx1 mice (not shown). ( $\mathbf{f}$ and $\mathbf{g}$ ) Tg-Trx1 mice and NTg littermates were subjected to either transverse aortic banding or sham operation. Aortic banding was applied for 2 weeks. (f) The effect of pressure overload on oxidative stress in the heart. The heart contents of MDA and MDA plus 4-HAE are shown. (g) LWW/BW (left) and percentage increase in LWW/BW in response to aortic banding (right) were determined. Similar results were obtained for LWW/TL (not shown). 


\section{Table 4}

Base-line echocardiographic data of Tg-Trx1 mice and NTg littermates at the age of 3 months

\begin{tabular}{lcc} 
& NTg $(n=8)$ & Tg-DN-Trx $1(n=7)$ \\
DSEP Wt $(\mathrm{mm})$ & $0.722 \pm 0.021$ & $0.725 \pm 0.013$ \\
LVEDD $(\mathrm{mm})$ & $3.852 \pm 0.038$ & $3.840 \pm 0.075$ \\
DP Wt $(\mathrm{mm})$ & $0.682 \pm 0.021$ & $0.713 \pm 0.023$ \\
SSEP Wt $(\mathrm{mm})$ & $1.150 \pm 0.025$ & $1.179 \pm 0.027$ \\
LVESD $(\mathrm{mm})$ & $2.446 \pm 0.040$ & $2.485 \pm 0.059$ \\
SP Wt $(\mathrm{mm})$ & $0.985 \pm 0.022$ & $1.024 \pm 0.029$ \\
EF & $0.741 \pm 0.013$ & $0.722 \pm 0.015$ \\
\%FS & $36.5 \pm 1.1$ & $35.5 \pm 1.6$ \\
HR (beats per min) & $402 \pm 15$ & $402 \pm 16$ \\
\hline
\end{tabular}

Data are mean \pm SEM.

Trx1 mice, because Tg-Trx1 mice exhibited opposite responses to pressure overload, namely reduced levels of cardiac hypertrophy and oxidative stress. Furthermore, downregulation of Trx1 by antisense plasmid and Morpholino oligo also caused cardiac hypertrophy in cultured cardiac myocytes, consistent with the notion that the Trx 1 activity is a critical regulator of cardiac hyper- trophy. It should be noted, however, that the contribution of the signaling mechanisms mediated by direct interaction with Trx1 to cardiac phenotype of Tg-DNTrx1 mice remains to be elucidated.

A search for downstream signaling mechanisms causing cardiac hypertrophy in Tg-DN-Trx1 mice indicated that the Ras-Raf-1-ERK pathway, rather than stressresponsive protein kinases, is activated in Tg-DN-Trx1 mice. A well-compensated concentric form of cardiac hypertrophy in $\mathrm{Tg}$-DN-Trx1 mice is similar to that in transgenic mice with cardiac-specific overexpression of MAP kinase kinase 1 (36). It has been shown that oxidation of Cys118 in Ras increases GTPase activity of Ras (19). In fact, our results indicated that Ras is S-thiolated in the presence of DN-hTrx1 or antisense Trx1.

It has been recently shown that mice deficient in $\operatorname{Tr} x 2$, a Trx1 homolog localized in mitochondria, are embryonic lethal because of massive apoptosis (37). Although Trx2 is expressed in the heart, we do not know whether the activity of $\operatorname{Trx} 2$ in mitochondria is affected in Tg-DN-Trx1 mice, because of technical difficulties in isolating $\operatorname{Tr} x 2$ from the heart samples. However, since the cellular effect of $\operatorname{Tr} \times 2$ is predominantly cell protec- a

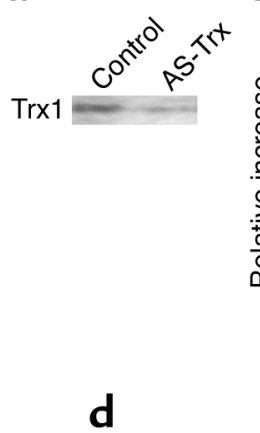

b

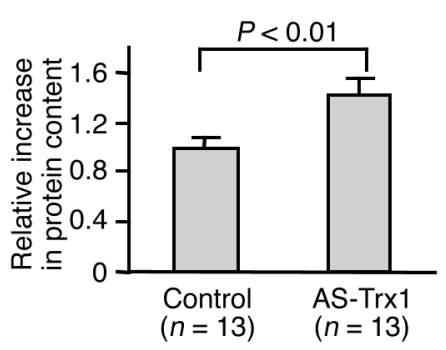

c

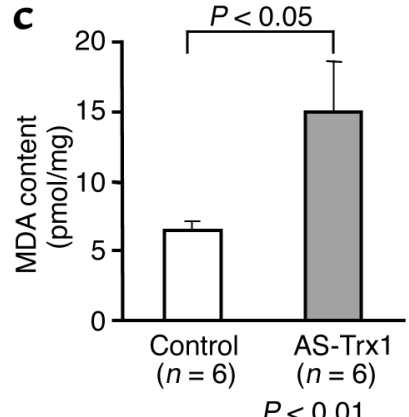

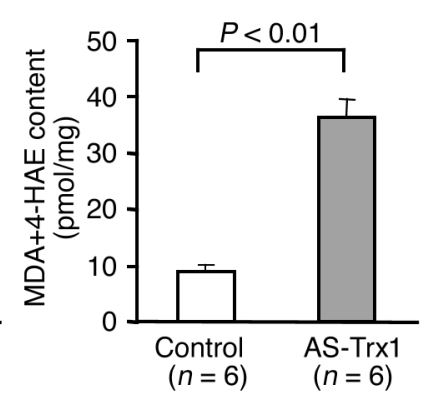

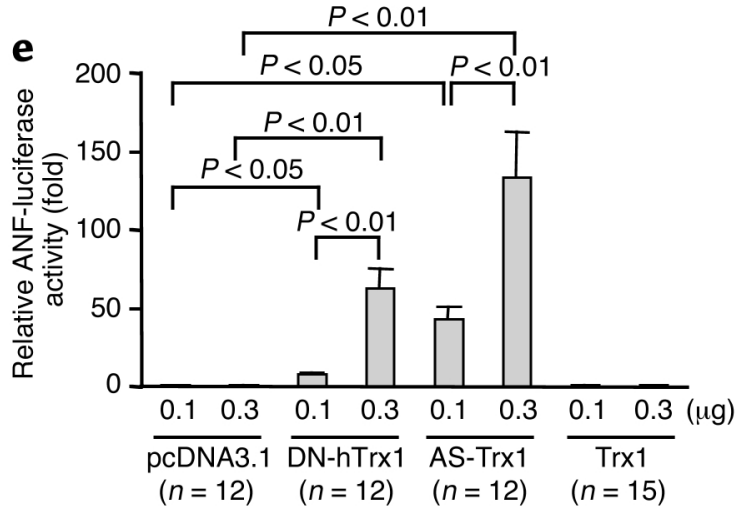

\section{Figure 8}

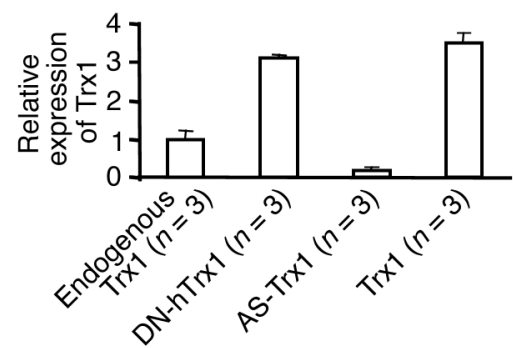

(a-c) Neonatal rat cardiac myocytes were treated with either control Morpholino oligo or Morpholino antisense oligo for rat Trx 1 ( $2 \mu \mathrm{M})$ using osmotic delivery. Myocytes were harvested 48 hours after application of the oligo. (a) Expression of Trx1 was determined by immunoblotting with anti-Trx1 Ab (4H9). (b) Total cardiac myocyte protein content was determined. The mean value in control Morpholino oligo-treated myocytes was designated as 1. (c) Cellular content of MDA and MDA plus 4-HAE was determined as described in Methods. (d) COS-7 cells grown in 60-mm dishes were transfected with either empty vector plasmid or pcDNA3.1 harboring DN-hTrx1, antisense rat Trx1 (AS-Trx1), or Trx1. Forty-eight hours after transfection, cells were harvested, and expression of Trx 1 was determined by immunoblot analysis. The level of Trx1 expression quantitated by densitometric analyses is shown. (e) Cardiac myocytes were transfected with ANF-luciferase (-638) (ANFluciferase reporter gene containing 638 base pairs upstream of the rat ANF gene transcription start site) and SV40- $\beta$-galactosidase, together with pcDNA3.1 alone (empty vector) or with pcDNA3.1 harboring DN-hTrx1, antisense rat Trx1, or Trx1. Forty-eight hours after transfection, activities of luciferase and $\beta$-galactosidase were determined. The activity of luciferase was normalized by that of $\beta$-galactosidase. 
tive (37), if the activity of $\operatorname{Tr} x 2$ is significantly inhibited, a well-compensated cardiac hypertrophy in Tg-DN-Trx1 mice would be unexpected. It would be interesting to generate mice with cardiac-specific deletion of Trx 2 or expression of dominant negative $\operatorname{Tr} \times 2$ and compare their phenotype with that of Tg-DN-Trx1 mice.

Recent evidence suggests that the activity of endogenous $\operatorname{Tr} x 1$ can be posttranslationally modified through interaction with other antioxidant mechanisms and oxidant species. For example, Cys 72 of Trx1 undergoes glutathiolation in response to oxidative stress, which abolishes the enzymatic activity of $\operatorname{Tr} 1$ (38). By contrast, nitric oxide S-nitrosylates Cys69 of Trx1, thereby stimulating the activity of $\operatorname{Tr} x 1$ (39). The activity of $\operatorname{Tr} x 1$ is also subjected to regulation by the Trx1-interacting proteins, such as VDUP1 (22). Thus, Tg-DN-Trx1 and TgTrx1 mice are useful to elucidate functional consequences of $\operatorname{Tr} x 1$ modulation in the heart in vivo.

In summary, our results suggest that endogenous Trx1 is an important antioxidant in the mouse heart, and that increases in oxidative stress caused by decreases in the Trx 1 activity stimulate a concentric form of cardiac hypertrophy in vivo. Thus, both $\operatorname{Trx} 1$ and its downstream target proteins may be an important therapeutic focus for treatment of cardiac hypertrophy.

\section{Acknowledgments}

We thank Jeffrey Robbins for providing us with the $\alpha$-myosin heavy chain promoter construct. We thank Daniela Zablocki and Dennis Quinio for critical reading of the manuscript. This work was supported in part by grants from the NIH (HL-59139, HL-33107, HL-33065, HL-65182, HL-65183, AG-14121, HL-69020, HL-67724, and HL-67727) and an Established Investigator Award from the American Heart Association (0340123N).

1. Sawyer, D.B., et al. 2002. Role of oxidative stress in myocardial hypertrophy and failure. J. Mol. Cell. Cardiol. 34:379-388.

2. Sorescu, D., and Griendling, K.K. 2002. Reactive oxygen species, mitochondria, and $\mathrm{NAD}(\mathrm{P}) \mathrm{H}$ oxidases in the development and progression of heart failure. Congest. Heart Fail. 8:132-140.

3. Nakamura, H., Nakamura, K., and Yodoi, J. 1997. Redox regulation of cellular activation. Annu. Rev. Immunol. 15:351-369.

4. Masutani, H., and Yodoi, J. 2002. Thioredoxin. Overview. Methods Enzymol. 347:279-286.

5. Kishimoto, C., et al. 2001. Serum thioredoxin (TRX) levels in patients with heart failure. Jpn. Circ. J. 65:491-494.

6. Shioji, K., et al. 2000. Upregulation of thioredoxin (TRX) expression in giant cell myocarditis in rats. FEBS Lett. 472:109-113.

7. Wang, Y., De Keulenaer, G.W., and Lee, R.T. 2002. Vitamin D(3)-up-regulated protein-1 is a stress-responsive gene that regulates cardiomyocyte viability through interaction with thioredoxin. J. Biol. Chem. 277:26496-26500.

8. Shioji, K., et al. 2002. Overexpression of thioredoxin-1 in transgenic mice attenuates adriamycin-induced cardiotoxicity. Circulation. 106:1403-1409.

9. Nakamura, H., Mitsui, A., and Yodoi, J. 2002. Thioredoxin overexpression in transgenic mice. Methods Enzymol. 347:436-440.

10. Gallegos, A., et al. 1996. Transfection with human thioredoxin increases cell proliferation and a dominant-negative mutant thioredoxin reverses the transformed phenotype of human breast cancer cells. Cancer Res. 56:5765-5770.

11. Esterbauer, H., Schaur, R.J., and Zollner, H. 1991. Chemistry and biochemistry of 4-hydroxynonenal: malonaldehyde and related aldehydes. Free Radic. Biol. Med. 11:81-128.

12. Liang, Q., et al. 2002. Overexpression of metallothionein reduces diabetic cardiomyopathy. Diabetes. 51:174-181.

13. Sadoshima, J., et al. 2002. The MEKK1-JNK pathway plays a protective role in pressure overload but does not mediate cardiac hypertrophy.
J. Clin. Invest. 110:271-279. doi:10.1172/JCI200214938.

14. Holmgren, A., and Bjornstedt, M. 1995. Thioredoxin and thioredoxin reductase. Methods Enzymol. 252:199-208.

15. Asai, K., et al. 1999. $\beta$-Adrenergic receptor blockade arrests myocyte damage and preserves cardiac function in the transgenic $\mathrm{G}_{\mathrm{s}} \alpha$ mouse. J. Clin. Invest. 104:551-558.

16. Date, M.O., et al. 2002. The antioxidant N-2-mercaptopropionyl glycine attenuates left ventricular hypertrophy in in vivo murine pressure-overload model. J. Am. Coll. Cardiol. 39:907-912.

17. Tomita, H., et al. 2003. Inducible cAMP early repressor (ICER) is a negative-feedback regulator of cardiac hypertrophy and an important mediator of cardiac myocyte apoptosis in response to beta-adrenergic receptor stimulation. Circ. Res. 93:12-22.

18. Sadoshima, J., and Izumo, S. 1995. Rapamycin selectively inhibits angiotensin II-induced increase in protein synthesis in cardiac myocytes in vitro. Potential role of 70-kD S6 kinase in angiotensin II-induced cardiac hypertrophy. Circ. Res. 77:1040-1052.

19. Lander, H.M., et al. 1996. Redox regulation of cell signalling. Nature. 381:380-381.

20. Liu, Y., and Min, W. 2002. Thioredoxin promotes ASK1 ubiquitination and degradation to inhibit ASK1-mediated apoptosis in a redox activityindependent manner. Circ. Res. 90:1259-1266.

21. Wakasugi, N., et al. 1990. Adult T-cell leukemia-derived factor/thioredoxin, produced by both human T-lymphotropic virus type I- and Epstein-Barr virus-transformed lymphocytes, acts as an autocrine growth factor and synergizes with interleukin 1 and interleukin 2. Proc. Natl. Acad. Sci. U. S. A. 87:8282-8286.

22. Schulze, P.C., De Keulenaer, G.W., Yoshioka, J., Kassik, K.A., and Lee, R.T. 2002. Vitamin D3-upregulated protein-1 (VDUP-1) regulates redoxdependent vascular smooth muscle cell proliferation through interaction with thioredoxin. Circ. Res. 91:689-695.

23. Eaton, P., Byers, H.L., Leeds, N., Ward, M.A., and Shattock, M.J. 2002. Detection, quantitation, purification, and identification of cardiac proteins S-thiolated during ischemia and reperfusion. J. Biol. Chem. 277:9806-9811.

24. Xie, Z., et al. 1999. Intracellular reactive oxygen species mediate the linkage of $\mathrm{Na}+\mathrm{K}+$-ATPase to hypertrophy and its marker genes in cardiac myocytes. J. Biol. Chem. 274:19323-19328.

25. Nakamura, K., et al. 1998. Inhibitory effects of antioxidants on neonatal rat cardiac myocyte hypertrophy induced by tumor necrosis factor-alpha and angiotensin II. Circulation. 98:794-799.

26. Amin, J.K., et al. 2001. Reactive oxygen species mediate alpha-adrenergic receptor-stimulated hypertrophy in adult rat ventricular myocytes. J. Mol. Cell. Cardiol. 33:131-139.

27. Siwik, D.A., et al. 1999. Inhibition of copper-zinc superoxide dismutase induces cell growth, hypertrophic phenotype, and apoptosis in neonatal rat cardiac myocytes in vitro. Circ. Res. 85:147-153.

28. Takagi, Y., et al. 1999. Overexpression of thioredoxin in transgenic mice attenuates focal ischemic brain damage. Proc. Natl. Acad. Sci. U. S. A. 96:4131-4136.

29. Saitoh, M., et al. 1998. Mammalian thioredoxin is a direct inhibitor of apoptosis signal-regulating kinase (ASK) 1. EMBO J. 17:2596-2606.

30. Watson, J.A., Rumsby, M.G., and Wolowacz, R.G. 1999. Phage display identifies thioredoxin and superoxide dismutase as novel protein kinase Cinteracting proteins: thioredoxin inhibits protein kinase C-mediated phosphorylation of histone. Biochem. J. 343:301-305.

31. Nishiyama, A., et al. 1999. Demonstration of the interaction of thioredoxin with p40phox, a phagocyte oxidase component, using a yeast twohybrid system. Immunol. Lett. 68:155-159.

32. Lee, S.R., et al. 2002. Reversible inactivation of the tumor suppressor PTEN by $\mathrm{H} 2 \mathrm{O} 2$. J. Biol. Chem. 277:20336-20342.

33. Hirota, K., et al. 1999. Distinct roles of thioredoxin in the cytoplasm and in the nucleus. A two-step mechanism of redox regulation of transcription factor NF-kappaB. J. Biol. Chem. 274:27891-27897.

34. Makino, Y., et al. 1999. Direct association with thioredoxin allows redox regulation of glucocorticoid receptor function. J. Biol. Chem. 274:3182-3188.

35. Ueno, M., et al. 1999. Thioredoxin-dependent redox regulation of p53mediated p21 activation. J. Biol. Chem. 274:35809-35815.

36. Bueno, O.F., et al. 2000. The MEK1-ERK1/2 signaling pathway promotes compensated cardiac hypertrophy in transgenic mice. EMBOJ 19:6341-6350

37. Nonn, L., Williams, R.R., Erickson, R.P., and Powis, G. 2003. The absence of mitochondrial thioredoxin 2 causes massive apoptosis, exencephaly, and early embryonic lethality in homozygous mice. Mol. Cell. Biol. 23:916-922.

38. Casagrande, S., et al. 2002. Glutathionylation of human thioredoxin: a possible crosstalk between the glutathione and thioredoxin systems. Proc. Natl. Acad. Sci. U. S. A. 99:9745-9749.

39. Haendeler, J., et al. 2002. Redox regulatory and anti-apoptotic functions of thioredoxin depend on S-nitrosylation at cysteine 69. Nat. Cell Biol. 4:743-749. 